\title{
ANÁLISE DA RESISTÊNCIA DE VIGAS DE CONCRETO ARMADO À FLEXÃO COM FIBRA DE CARBONO PELO MÉTODO NEAR SURFACE MOUNTED (NSM)
}

\author{
PINTO, LAURA GOMES \\ Mestranda em Construção Civil e Estruturas \\ Universidade de Brasília \\ Distrito Federal; Brasil \\ laura.gomesp@gmail.com \\ RODRIGES, GEORGE WILSON ALBERNAZ \\ Graduando em Engenharia Civil \\ Universidade de Brasília \\ Distrito Federal; Brasil \\ georgealbernaz@gmail.com
}

\author{
DAL PONT, RENATA SOARES PIAZZA \\ Mestranda em Construção Civil e Estruturas \\ Universidade de Brasília \\ Distrito Federal; Brasil \\ renatapiazza3@hotmail.com
}

\author{
OLIVEIRA, MARCOS HONORATO \\ Professor \\ Universidade de Brasília \\ Distrito Federal; Brasil \\ honorato.eng@gmail.com
}

\section{RESUMO}

Ao longo dos anos a sociedade vem ampliando o uso de estruturas de concreto armado. Concomitante a isso, essas estruturas estão sujeitas a processos de deteriorização natural e acelerada, proporcionada pela negligência e falta de manutenção. Dessa maneira, o reforço desses elementos de concreto armado torna-se necessário, sendo altamente promissor o desenvolvimento e aperfeiçoamento dos métodos de reforço estrutural. Dentre as técnicas de reforço estrutural, destaca-se a que utiliza polímeros reforçados com fibras (PRF) e os principais sistemas compostos que aplicam PRF são o Externally Bonded Reinforcement (EBR) e o Near Surface Mounted (NSM). A técnica NSM consiste em instalar barras ou lâminas em ranhuras encravadas na superfície do elemento estrutural e preenchê-las totalmente com resina epóxi. Este artigo tem como objetivo analisar o comportamento de vigas de concreto armado reforçadas à flexão com Polímeros Reforçados com Fibras de Carbono (PRFC) em forma de lâminas, utilizando a técnica NSM. É avaliado o desempenho das recomendações normativas ACI 440.2R (2017) e fib Bulletin 14 (2001) por meio de uma análise comparativa dos resultados teóricos com os resultados experimentais de um banco de dados compostas por vigas reforçadas à flexão com fibras de carbono utilizando a técnica NSM ensaiadas por diversos autores.

Palavras-chave: Near Surface Mounted (NSM), Fibras de Carbono, Flexão,Vigas de Concreto Armado, Reforço.

\begin{abstract}
Over the years society has been expanding the use of reinforced concrete structures. Concomitant to this fact, these structures are subject to processes of natural and accelerated deterioration as a result of negligence and lack of maintenance. Hence, strengthening these reinforced concrete elements becomes necessary, and the development and improvement of structural rehabilitation methods is highly promising. Among the techniques of structural strengthening, the one which uses Fiber Reinforced Polymers (FRP) stands out, and the main composite systems that apply FRP are Externally Bonded Reinforcement (EBR) and Near Surface Mounted (NSM). The NSM technique consists of installing bars or blades in grooved grooves on the surface of the structural element and filling them completely with epoxy resin. This paper aims to analyze the behavior of reinforced concrete beams strengthened with blades of Carbon Fiber Reinforced Polymers (CFRP) to resist flexural stresses, using the NSM technique. The performance of the standards ACI 440.2R (2017) and fib Bulletin 14 (2001) are analyzed by comparing theoretical estimates of the resistance with experimental results of a database created from results of experimental tests performed by several authors in reinforced concrete beams strengthened with carbon fibers, using the NSM technique, which were subjected to flexural loads. Keywords: Near Surface Mounted (NSM), Carbon Fyber, Flexion, Reinforced Concrete Beams.
\end{abstract}




\section{INTRODUÇÃO}

Na sociedade contemporânea, o concreto é o material mais consumido na construção civil e tende a se manter nessa posição no mundo por um considerável período de tempo, sendo atualmente o segundo material mais utilizado no mundo, ficando atrás apenas da água. Como apresentado por Machado e Machado (2015), o concreto é um material que, assim como qualquer outro, está sujeito à ação deletéria do meio ambiente e das agressões físicas e químicas. Assim, o processo de enfraquecimento e desgaste de uma estrutura de concreto é natural e, a fim de garantir a sua vida útil, a estrutura necessita de manutenção regular e reparos. Tem-se, dessa maneira, perante a necessidade verificada de reabilitação de grande parte do sistema estrutural, deve-se compreender quando uma estrutura precisa ser reforçada.

O reforço caracteriza-se como uma atividade específica para os casos em que se deseja elevar o desempenho de uma estrutura, dotando-a de maior resistência e/ou rigidez. Diante de diversas causas que podem levar à perda de resistência de uma estrutura, foram desenvolvidas diversas técnicas e utilizados os mais variados materiais para a execução do reforço estrutural ao longo da história. Um desses materiais consiste no uso de polímeros reforçados com fibras (PRF), um material compósito em que o componente estrutural é dado pela união entre fibras contínuas de diversos polímeros, tratando-se de um material não homogêneo, anisotrópico, o qual apresenta um comportamento elástico linear até a ruptura. A utilização de fibras como componente estrutural do reforço soluciona o problema da corrosão do material, além de serem materiais muito leves, com excelentes propriedades mecânicas e de fácil aplicação e adaptação às superfícies. Atualmente tem ganhado destaque o polímero reforçado com fibra de carbono (PRFC), devido a elevada resistência apresentada por este material em comparação com as demais fibras (Machado e Machado, 2015). Assim, este trabalho analisará os casos de reforço estrutural com a utilização de polímeros reforçados com fibras de carbono (PRFC).

Além de identificar a necessidade de reforço de uma estrutura e optar pelo material a ser utilizado, deve-se determinar qual a melhor técnica de aplicação do reforço a ser realizada. Dentre as diversas técnicas de reforço estrutural que surgiram com a utilização de polímero reforçado com fibra de carbono, podemos citar duas principais: o Externally Bonded Reinforcement (EBR) e a sua variação, o Near Surface Mounted (NSM). A técnica EBR consiste em colar as fibras externamente ao elemento estrutural que será reforçado, sendo comum a utilização de materiais compósitos na forma de laminados ou mantas. A colagem deste material é realizada com a utilização de uma resina específica, geralmente à base de epóxi. Já a técnica NSM consiste na instalação de barras ou lâminas de PRF em ranhuras cravadas longitudinalmente na superfície do elemento reforçado. Esse material é fixado com a utilização da mesma resina aplicada na técnica anterior, a qual também é utilizada para preencher a ranhura após a instalação do reforço, conforme mostra a Figura 1.

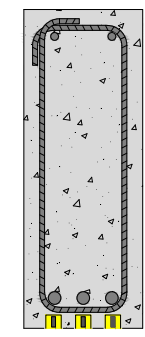

Figura 1: Viga reforçada pelo método NSM.

Comparando a eficiência das técnicas de reforço com PRF, percebe-se uma vantagem na aplicação do NSM, como observado por Coelho et al (2011), que mostrou que o NSM é mais eficiente que os demais métodos. É apresentado nesse trabalho que para um mesmo deslocamento aplicado em vigas reforçadas por diferentes métodos de reforço estrutural, os elementos que utilizam a técnica NSM suportam uma força superior antes de atingirem a ruptura do que aqueles reforçados com EBR. Segundo Machado e Machado (2015), esse comportamento pode ser justificado pela maior eficiência construtiva da técnica NSM, uma vez que nesse caso os esforços nas fibras são incorporados de forma mais efetiva ao concreto por meio da transferência de esforços, via resina, para as superfícies das ranhuras criadas entre a superfície externa do concreto e os estribos existentes. Dessa forma, neste trabalho serão abordados os elementos reforçados pelo método Near Surface Mounted (NSM).

Uma das motivações deste trabalho consiste no fato de não existirem recomendações normativas brasileiras sobre essa temática, sendo a sua análise uma fonte de estudo promissora e atual. Dessa forma, esta pesquisa trata da elaboração de um banco de dados com análises paramétricas de elementos reforçados com lâminas de carbono pela técnica NSM, trazendo mais informação ao meio técnico-científico brasileiro a respeito dessa interessante técnica de reforço. 
Tem-se, como objetivos deste trabalho, elaborar e analisar um banco de dados com resultados de ensaios experimentais em vigas reforçadas à flexão com fibras de carbono utilizando a técnica NSM de diversos autores disponíveis na literatura. Além disso, será calculado e analisado estatisticamente os resultados experimentais desse banco de dados pelas prescrições das normas ACI 440.2R (2017) e fib Bulletin 14 (2001), que tratam do dimensionamento de reforço estrutural com uso de PRFC. Será também avaliado o desempenho das recomendações normativas citadas por meio de uma análise comparativa dos resultados teóricos das estimativas de resistência com os resultados experimentais do banco de dados, verificando assim, a eficácia e precisão do uso das formulações propostas por essas recomendações normativas

\section{MÉTODOS DE CÁLCULO}

\subsection{ACI 440.2R}

O ACI 440.2R (2017) é uma norma norte-americana que apresenta recomendações para o dimensionamento de reforço utilizando polímeros reforçados com fibras de carbono (PRFC), buscando evitar modos de ruptura prematuros e sem avisos. Essa recomendação afirma que, para ser reforçado com PRF, o concreto deve possuir resistência superior à 17 MPa.

Para calcular a resistência à flexão de uma viga reforçada com PRFC pelo NSM deve-se, primeiramente, estimar a altura da linha neutra do elemento. Com isso pode-se estimar as deformações de cada parcela de força envolvida no mecanismo de ruptura da peça submetida à flexão, a partir da compatibilidade entre as deformações desenvolvidas em cada material da seção transversal do elemento fletido. A equação abaixo determina o valor de deformação do PRFC a partir da compatibilidade de deformações.

$$
\varepsilon_{f e}=\varepsilon_{c u} \cdot\left(\frac{n-x}{x}\right)-\varepsilon_{b i}<\varepsilon_{f d}
$$

Onde:

$\varepsilon_{f e}$ é a deformação efetiva no PRFC;

$\varepsilon_{c u}$ é a deformação de ruptura do concreto (igual à 0,003 para o ACI 440.2R);

$h$ é a altura da viga;

$x$ é a posição da linha neutra;

$\varepsilon_{b i}$ é a deformação encontrada no cobrimento da armadura de tração na viga antes do reforço.

Como o trabalho é voltado para a verificação da capacidade de carga de vigas ensaiadas à flexão, busca-se simular, pelo procedimento de cálculo, o estado limite último real, com os valores de tensão e deformação efetivamente atuantes nos elementos. Assim, para a deformação efetiva na fibra serão utilizados valores presentes no banco de dados que foram coletados por meio de extensômetros instalados no PRF das vigas ensaiadas.

Como assume-se que não há pré-fissuração nos elementos fletidos ensaiados e que, consequentemente, $\varepsilon_{b i}=0$, é possível calcular a tensão na fibra a partir do valor de deformação efetiva obtido. Para isso, utiliza-se a Equação II, a qual baseiase na Lei de Hooke, visto que o ACI 440.2R (2017) considera um comportamento linear elástico até a ruptura para o material.

$$
f_{f e}=E_{f} \cdot \varepsilon_{f e}
$$

(Equação II)

Durante o dimensionamento, a definição da deformação efetiva indica o modo de ruptura. Como a deformação da fibra foi limitada, o ACI 440.2R considera que não ocorrerá o modo de ruptura da fibra (RF). Pelo equilíbrio das deformações e a Lei de Hooke, temos:

$$
x=\frac{\left(A_{s} \cdot f_{s}\right)+\left(A_{f} \cdot f_{f e}\right)-\left(A_{s}^{\prime} \cdot f_{s}^{\prime}\right)}{\beta_{1} \cdot \alpha_{1} \cdot f_{c} \cdot b_{w}}
$$

Onde:

$A_{s}$ é a área de aço da armadura inferior;

$A_{f}$ é a área do reforço aplicado na viga; 
$A_{s}$ 'é a área de aço da armadura superior;

$f_{s}$ é a tensão no aço da armadura inferior;

$f_{s}$ ' é a tensão no aço da armadura superior;

$f_{f e}$ é a tensão efetiva do PRFC;

$f_{c}$ é a resistência à compressão do concreto;

$b_{w}$ é a largura da viga;

$\alpha_{l}$ é o fator multiplicador do $\mathrm{f}_{\mathrm{c}}{ }^{\prime}$ para determinar a intensidade de um bloco retangular de distribuição de tensões do concreto equivalente;

$\beta_{1}$ é o coeficiente que determina a aproximação da curva da resultante de compressão do concreto a um retângulo.

A determinação da altura da linha neutra pode ser realizada com um cálculo iterativo, onde deve-se buscar um valor para $x$ onde o somatório das forças de tração e de compressão atuantes na seção transversal do elemento seja zero. O ACI 440.2R (2017) recomenda o método de tentativas e erros para verificar o posicionamento da linha neutra, sendo possível estabelecer uma correlação entre a posição dessa linha e o somatório das componentes das forças resistentes na seção transversal da viga, a partir de um processo iterativo. Por fim, com a posição da linha neutra corrigida é possível determinar o momento resistente $\left(\mathrm{M}_{\mathrm{R}}\right)$ da viga reforçada a partir da Equação IV.

$$
M_{R}=A_{s} \cdot f_{s} \cdot\left(d-\frac{\beta_{1} \cdot x}{2}\right)+0,85 \cdot A_{f} \cdot f_{f e} \cdot\left(h-\frac{\beta_{1} \cdot x}{2}\right)+A_{s}^{\prime} \cdot f_{s}^{\prime} \cdot\left(\frac{\beta_{1} \cdot x}{2}-d^{\prime}\right)
$$

(Equação IV)

Nessa equação, o fator 0,85 que multiplica o termo de contribuição da fibra corresponde a um termo de minoração da força do PRF, denominado $\psi$ f e definido pelo ACI 440.2R (2017).

\section{2 fib Bulletin 14}

O fib Bulletin 14 (2001) traz recomendações para o dimensionamento de vigas reforçadas com PRF aderido externamente pelo método EBR, sem tratar da aplicação do NSM. Porém, algumas de suas formulações podem ser adaptadas, possibilitando o cálculo da capacidade resistiva para este caso.

Dessa forma, para a determinação do momento resistente das vigas reforçadas pelo NSM considerando a atuação completa dos compósitos, serão utilizadas as mesmas formulações apresentadas pelo código europeu para o EBR. Assim como o ACI 440.2R (2017), o fib Bulletin 14 (2001) propõem uma limitação do valor de deformação de projeto do reforço, como mostra a Equação V:

Onde:

$$
\varepsilon_{f d}=\frac{\varepsilon_{f u}}{\gamma_{f}}
$$

(Equação V)

$\varepsilon_{f d}$ é o valor limite de deformação da fibra a ser adotado no dimensionamento e verificação do reforço para evitar rupturas prematuras da fibra;

$\varepsilon_{f u}$ é a deformação última observada na fibra no momento da ruptura;

$\gamma_{f}$ é o fator de segurança, dependendo da condição de trabalho.

A partir da deformação de projeto e do equilíbrio de deformações da seção da viga analisada, o fib Bulletin 14 (2001) estabelece o valor da deformação efetiva da fibra, como mostra a Equação VI abaixo:

$$
\varepsilon_{f e}=\varepsilon_{c u} \cdot\left(\frac{h-x}{x}\right)-\varepsilon_{b i}<\varepsilon_{f d}
$$

Onde:

$\varepsilon_{f e}$ é a deformação efetiva no PRFC; 
$\varepsilon_{c u}$ é a deformação de ruptura do concreto;

$h$ é a altura da viga;

$x$ é a posição da linha neutra;

$\varepsilon_{b i}$ é a deformação encontrada no cobrimento da armadura de tração na viga antes do reforço.

Pela análise desses valores de deformação calculados é possível obter o modo de ruptura previsto. Com os valores de deformação efetiva da fibra é possível compará-los ao valor de deformação última, a fim de verificar se ocorrerá uma ruptura frágil dada pela ruptura do PRF. Ainda se verifica se ocorre esmagamento do concreto pela análise do valor de deformação do concreto, que ao ultrapassar a deformação última adotada pela norma $(0,0035)$, acarreta em uma ruptura com esmagamento do concreto. Nos demais casos, deve-se observar uma delaminação do concreto ou descolamento do PRF.

Com as tensões atuantes no elemento, deve-se proceder ao cálculo da altura da linha neutra x da seção analisada, de forma análoga ao realizado pelo ACI 440.2R (2017), como mostra a Equação VII.

$$
x=\frac{\left(A_{s} \cdot f_{s}\right)+\left(A_{f} \cdot f_{f e}\right)-\left(A_{s}^{\prime} \cdot f_{s}^{\prime}\right)}{0,85 \cdot \Psi \cdot f_{c} \cdot b_{w}}
$$

(Equação VII)

\section{Onde:}

$A_{s}$ é a área de aço da armadura inferior;

$A_{f}$ é a área do reforço aplicado na viga;

$A_{s}$ ' é a área de aço da armadura superior;

$f_{s}$ é a tensão no aço da armadura inferior;

$f_{s}$ ' é a tensão no aço da armadura superior;

$f_{f e}$ é a tensão efetiva do PRFC;

$f_{c}$ é a resistência à compressão do concreto;

$b_{w}$ é a largura da viga;

$\Psi$ é o coeficiente que determina a aproximação da curva da resultante de compressão do concreto a um retângulo.

Com a determinação da altura da linha neutra, procede-se à determinação do momento resistente da seção, dado pela Equação VIII abaixo:

$$
M_{R}=A_{s} \cdot f_{s} \cdot\left(d-\delta_{g} \cdot x\right)+0,85 \cdot A_{f} \cdot f_{f e} \cdot\left(h-\delta_{g} \cdot x\right)+A_{s}^{\prime} \cdot f_{s}^{\prime} \cdot\left(\delta_{g} \cdot x-d^{\prime}\right)
$$

Onde:

$\delta g$ é o coeficiente que determina a aproximação da curva da resultante de compressão do concreto a um retângulo, sendo 0,4 para rupturas que consideram o escoamento do concreto após o escoamento da armadura (CC) e para a ruptura do compósito após o escoamento da armadura (RF)

\section{METODOLOGIA}

A fim de avaliar as recomendações normativas que regem o cálculo do reforço estrutural aderido externamente ao elemento, foi elaborado um banco de dados com informações recolhidas da literatura sobre vigas de concreto armado reforçadas à flexão com lâminas de carbono pelo método NSM, sendo selecionadas apenas as vigas ensaiadas pelo método de Sttutgart, que romperam por flexão. As Tabela 1 e 2 apresentam um resumo das características dos 49 espécimes de 12 autores diferentes contidos no banco de dados. 


\section{CBPAT 2020

Tabela 1 - Características das vigas do banco de dados

\begin{tabular}{|c|c|c|c|c|c|c|c|c|c|c|}
\hline Autor & $\begin{array}{l}\mathrm{N}^{\circ} \text { de } \\
\text { vigas }\end{array}$ & $\begin{array}{c}\mathrm{b}_{\mathrm{w}} \\
(\mathrm{mm})\end{array}$ & $\begin{array}{c}\mathrm{h} \\
(\mathrm{mm})\end{array}$ & $\underset{(\mathrm{mm})}{\mathrm{d}}$ & $\begin{array}{c}\mathrm{d}^{\prime} \\
(\mathrm{mm})\end{array}$ & $\begin{array}{c}\mathrm{a} \\
(\mathrm{mm})\end{array}$ & $\begin{array}{c}\mathrm{As}_{\mathrm{S}} \\
\left(\mathrm{mm}^{2}\right)\end{array}$ & $\begin{array}{c}\mathrm{As}^{\prime} \\
\left(\mathrm{mm}^{2}\right)\end{array}$ & $\begin{array}{c}\mathrm{f}_{\mathrm{ys}} \\
(\mathrm{MPa})\end{array}$ & $\begin{array}{c}\mathrm{f}_{\mathrm{c}} \\
(\mathrm{MPa})\end{array}$ \\
\hline $\begin{array}{l}\text { Fortes } e t \\
\text { al (2002) }\end{array}$ & 4 & 100 & $170-180$ & 149-159 & 21 & 500 & $57-160$ & 101 & $\begin{array}{r}450- \\
750\end{array}$ & 46,1 \\
\hline $\begin{array}{c}\text { Dias et al } \\
\text { (2012) }\end{array}$ & 3 & 150 & 300 & 276 & 14 & 900 & 157 & 101 & $\begin{array}{l}535- \\
567\end{array}$ & 50,2 \\
\hline $\begin{array}{l}\text { Barros et } \\
\text { al (2007) }\end{array}$ & 3 & 120 & 170 & $142-146$ & 29 & 300 & $39-100$ & 66 & $\begin{array}{c}627- \\
788\end{array}$ & 44,2 \\
\hline $\begin{array}{l}\text { Billota } e t \\
\text { al (2015) }\end{array}$ & 2 & 120 & 160 & 115 & 30 & 925 & 157 & 157 & 540 & 16,8 \\
\hline $\begin{array}{c}\text { Kang et } \\
\text { al (2005) }\end{array}$ & 4 & 200 & 300 & $265-275$ & $26,5-37$ & 1050 & 235,6 & 398 & $\begin{array}{c}430- \\
480 \\
\end{array}$ & 31,5 \\
\hline $\begin{array}{c}\text { Seo et al } \\
(2016)\end{array}$ & 2 & 200 & 400 & 360 & 40 & 850 & 314,2 & 236 & 486,7 & 21 \\
\hline $\begin{array}{c}\text { Lim } \\
(2013)\end{array}$ & 6 & 200 & 300 & 260 & 38 & 825 & 508,9 & 265 & 500 & 30 \\
\hline $\begin{array}{l}\text { Moon et } \\
\text { al (2005) }\end{array}$ & 6 & 250 & 400 & 342 & 48 & 1270 & $\begin{array}{c}265,5- \\
398,2\end{array}$ & 157 & 600 & 30 \\
\hline $\begin{array}{c}\text { Yost et al } \\
(2007)\end{array}$ & 12 & $\begin{array}{c}152,4- \\
305\end{array}$ & 190 & $\begin{array}{l}144- \\
145,5 \\
\end{array}$ & - & 1219 & $\begin{array}{c}398,2- \\
402,1 \\
\end{array}$ & - & $\begin{array}{c}490- \\
510 \\
\end{array}$ & 37,2 \\
\hline $\begin{array}{l}\text { Coelho et } \\
\text { al (2011) }\end{array}$ & 1 & 200 & 300 & 269 & 31 & 900 & 235,6 & 157 & 455 & 53,1 \\
\hline $\begin{array}{c}\text { Ibrahim } \\
\text { (2014) }\end{array}$ & 3 & 200 & 400 & 372 & 28 & 600 & 157 & 157 & 560 & 25 \\
\hline $\begin{array}{c}\text { Kim } \\
(2015)\end{array}$ & 3 & 100 & 165 & 138 & - & $\begin{array}{l}328- \\
728\end{array}$ & 141,8 & - & 414 & 23 \\
\hline
\end{tabular}

Tabela 2 - Variáveis da viga de reforço

\begin{tabular}{|c|c|c|c|c|c|c|c|}
\hline Autor & $\begin{array}{c}\mathrm{N}^{\circ} \text { de } \\
\text { vigas }\end{array}$ & $\begin{array}{c}\mathrm{N}^{\circ} \mathrm{de} \\
\text { tiras }\end{array}$ & $\begin{array}{c}\mathrm{b}_{\mathrm{f}} \\
(\mathrm{mm})\end{array}$ & $\begin{array}{c}\mathrm{t}_{\mathrm{f}} \\
(\mathrm{mm})\end{array}$ & $\begin{array}{c}\mathrm{A}_{\mathrm{f}} \\
\left(\mathrm{mm}^{2}\right)\end{array}$ & $\begin{array}{c}\mathrm{E}_{\mathrm{f}} \\
(\mathrm{GPa})\end{array}$ & $\begin{array}{c}\varepsilon_{\mathrm{fu}} \\
(\%)\end{array}$ \\
\hline Fortes et al (2002) & 4 & $1-3$ & 10 & 1,47 & $15-44$ & 150 & 17 \\
\hline Dias et al $(2012)$ & 3 & $1-3$ & 10 & 1,40 & $14-42$ & 175 & 18 \\
\hline Barros et al (2007) & 3 & $1-3$ & 10 & 1,40 & $14-42$ & 159 & 14 \\
\hline Billota et al (2015) & 2 & $2-3$ & 10 & 1,40 & $28-42$ & 171 & 12 \\
\hline Kang et al (2005) & 4 & $1-4$ & $15-25$ & 1,2 & $18-120$ & 165 & 15 \\
\hline Seo et al (2016) & 2 & $1-3$ & 16 & $1,2-3,6$ & 58 & 160 & 17,5 \\
\hline Lim (2013) & 6 & $1-2$ & 30 & 6 & $180-360$ & 165 & 16,9 \\
\hline Moon et al $(2005)$ & 6 & $1-2$ & 25 & 1,2 & $30-60$ & 165 & 19 \\
\hline Yost et al (2007) & 12 & $1-2$ & 15 & 2,5 & $38-75$ & 136 & 12 \\
\hline $\begin{array}{c}\text { Coelho } \text { et al } \\
(2011)\end{array}$ & 1 & 4 & 15 & 1,41 & 85 & 158 & 15 \\
\hline Ibrahim $(2014)$ & 3 & 2 & $25-50$ & 1,2 & $60-120$ & 165 & 14 \\
\hline Kim (2015) & 3 & 1 & 16 & 2 & 32 & 124 & 17 \\
\hline
\end{tabular}

Com os dados contidos no banco de dados, realizou-se o cálculo do momento último de ruptura à flexão ( $\left.\mathrm{M}_{\mathrm{u}}\right)$ para as vigas de concreto armado reforçadas e comparou-se o seu resultado com o valor momento último obtido experimentalmente. Para o cálculo das vigas reforçadas com fibra de carbono foram utilizadas duas recomendações internacionais, o ACI 440.2R (2017) e o fib Bulletin 14 (2001). 


\section{RESULTADOS E DISCUSSÕES}

A fim de comparar os valores de momento resistente previsto $\left(M_{R}\right)$ com os de momento de ruptura experimental $\left(M_{u}\right)$ foram elaborados os gráficos para, como mostram as Figuras 2 e 3. A linha contínua presente nos gráficos representa a razão entre o momento resistente previsto e o momento de ruptura experimental igual a 1, portando os valores abaixo dessa linha são previsões contra à segurança e os valores acima dessa linha são a favor da segurança. A linha tracejada é uma regressão linear dos resultados obtidos, a qual fornece um valor de $\mathrm{R}^{2}$ para cada gráfico e permite analisar o desempenho de cada método analisado.

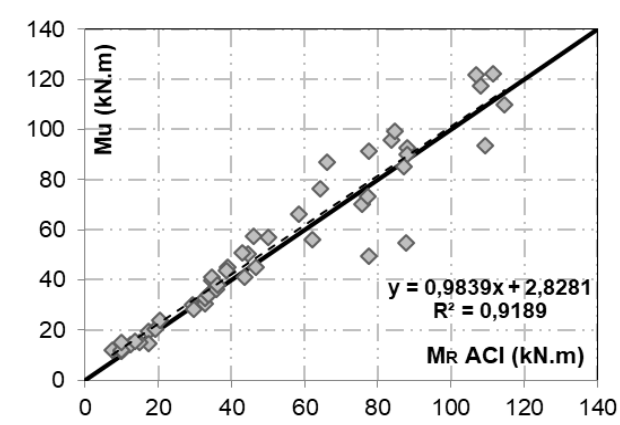

Figura 2: Análise da precisão do modelo do ACI 440.2R (2017)

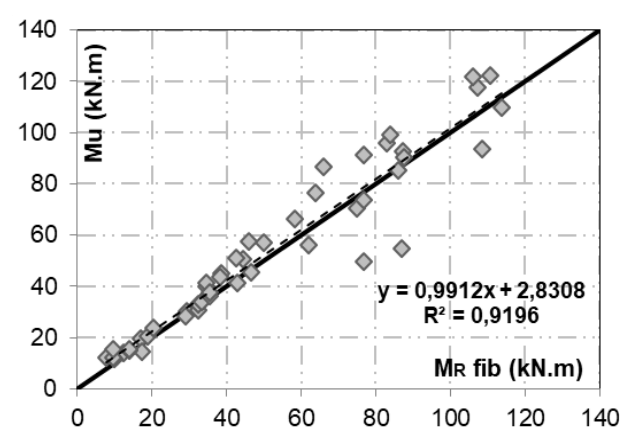

Figura 3: Análise da precisão do modelo do fib Bulletin $14(2001)$

A partir desses gráficos observa-se que os resultados apresentados pelo ACI 440.2R (2017) mostraram-se mais precisos e mais acurados que aqueles apresentados pelo fib Bulletin 14 (2001). Observa-se também que para momentos resistentes inferiores à $50 \mathrm{kN} . \mathrm{m}$, a razão entre o momento teórico e o resistente fica próxima ao valor unitário, enquanto que para valores maiores de momento resistente, a incerteza dada pelos resultados obtidos também aumenta.

Além disso, foi realizada uma análise de conformidade dos modos de ruptura, comparando os modos de ruptura teóricos com os modos de ruptura observados experimentalmente, com a finalidade de observar se as prescrições de cálculo analisadas previram de forma correta o modo de ruptura das vigas. A Tabela 3 apresenta o percentual de modos de ruptura previstos de forma correta por cada normativa de cálculo.

Tabela 3 - Conformidade dos modos de ruptura

\begin{tabular}{|c|c|c|c|}
\hline \multicolumn{4}{|c|}{ Conformidade Geral } \\
\hline \multicolumn{2}{|c|}{ ACI 440.2R (2017) } & \multicolumn{2}{|c|}{ fib Bulletin 14 (2001) } \\
\hline \multicolumn{2}{|c|}{$69,39 \%$} & \multicolumn{2}{|c|}{$55,10 \%$} \\
\hline Modo de ruptura & $\begin{array}{c}\text { Conformidade por modo } \\
\text { de ruptura }\end{array}$ & Modo de ruptura & $\begin{array}{c}\text { Conformidade por modo } \\
\text { de ruptura }\end{array}$ \\
\hline $\mathrm{DE} / \mathrm{FL}$ & $90,48 \%$ & $\mathrm{DE} / \mathrm{FL}$ & $95,24 \%$ \\
\hline $\mathrm{CC}$ & $56,00 \%$ & $\mathrm{CC}$ & $20,00 \%$ \\
\hline $\mathrm{RF}$ & $66,67 \%$ & RF & $66,67 \%$ \\
\hline \multicolumn{4}{|c|}{$\begin{array}{l}\text { Obs: } \\
\text { FL - Ruptura por delaminação do cobrimento. } \\
\text { DE- Ruptura por descolamento do PRF do substrato. } \\
\text { CC- Ruptura por esmagamento do concreto antes do escoamento do aço ou ruptura por escoamento do aço seguido pelo esmagamento do } \\
\text { concreto. }\end{array}$} \\
\hline
\end{tabular}

Assim, observa-se que as previsões dos modos de ruptura de ambas as recomendações normativas foram semelhantes, com exceção do modo de ruptura $\mathrm{CC}$, que apresentou resultados insatisfatórios de conformidade, principalmente para o fib Bulletin 14 (apenas 20\%). Além disso, observa-se que para o modo de ruptura DE/FL as previsões mostraram-se muito melhores, com taxas de conformidade superiores a $90 \%$ para as duas prescrições avaliadas. 
Também foi feita uma análise geral de alguns parâmetros estatísticos do banco de dados, como a média da razão $\mathbf{M}_{u} / \mathrm{M}_{\mathrm{R}}$, o seu desvio padrão e coeficiente de variação, além da quantificação dos resultados contra a segurança, ou seja, aqueles com relação $\mathrm{M}_{\mathrm{u}} / \mathrm{M}_{\mathrm{R}}$ inferior a 0,85 . Estes dados são apresentados na Tabela 4.

Tabela 4 - Parâmetros estatísticos do banco de dados geral

\begin{tabular}{|c|c|c|c|c|c|}
\hline Recomendações & $\begin{array}{c}\text { Qt. de } \\
\text { espécimes }\end{array}$ & Média & $\begin{array}{c}\text { Desvio Padrão } \\
(\%)\end{array}$ & $\begin{array}{c}\text { Coeficiente de } \\
\text { Variação (\%) }\end{array}$ & $\begin{array}{c}\text { Resultados Contra a } \\
\text { Segurança (\%) }\end{array}$ \\
\hline ACI 440.2R & 49 & 1,076 & 17,04 & 15,83 & 6,12 \\
\hline fib Bulletin 14 & 49 & 1,085 & 17,10 & 15,76 & 6,12 \\
\hline
\end{tabular}

A partir dos valores apresentados na Tabela 4, percebe-se que as duas recomendações de cálculo apresentaram valores bastante semelhantes. Ambas apresentaram médias superiores a 1 e apenas 6,12\% dos resultados contra a segurança e, assim, pode-se dizer que ambas se mostraram adequadas no quesito de segurança estrutural.

Por outro lado, os valores de desvio padrão e do coeficiente de variação se mostraram um pouco elevados, o que se deve principalmente à variação entre os modos de ruptura dos espécimes analisados e à maior incerteza dos resultados apresentados pelas vigas com maior momento resistente, como observado anteriormente. Por este motivo, a fim de refinar estas análises e comparar vigas que apresentem características mais semelhantes entre si, o banco de dados foi divido em três grupos de análise, sendo estes de acordo com o modo de ruptura de cada viga (CC, DE/FL e RF).

A Tabela 5 apresenta os grupos e as suas respectivas quantidades de espécimes, de acordo com cada normativa de cálculo analisada, além da média, desvio padrão, coeficiente de variação e percentual de resultados contra a segurança de cada grupo.

Tabela 5 - Parâmetros estatísticos do banco de dados por grupo

\begin{tabular}{|c|c|c|c|c|c|}
\hline \multicolumn{6}{|c|}{ Grupo CC } \\
\hline Recomendações & $\begin{array}{c}\text { Qt. de } \\
\text { espécimes }\end{array}$ & Média & $\begin{array}{c}\text { Desvio Padrão } \\
(\%)\end{array}$ & $\begin{array}{l}\text { Coeficiente de } \\
\text { Variação (\%) } \\
\end{array}$ & $\begin{array}{c}\text { Resultados Contra a } \\
\text { Segurança }\end{array}$ \\
\hline ACI $440.2 \mathrm{R}$ & 15 & 1,01 & 12,57 & $12,51 \%$ & $6,67 \%$ \\
\hline fib Bulletin 14 & 6 & 1,05 & 7,94 & $7,55 \%$ & $0,00 \%$ \\
\hline \multicolumn{6}{|c|}{ Grupo DE/FL } \\
\hline Recomendações & $\begin{array}{c}\text { Qt. de } \\
\text { espécimes }\end{array}$ & Média & $\begin{array}{c}\text { Desvio Padrão } \\
(\%)\end{array}$ & $\begin{array}{l}\text { Coeficiente de } \\
\text { Variação (\%) } \\
\end{array}$ & $\begin{array}{c}\text { Resultados Contra a } \\
\text { Segurança }\end{array}$ \\
\hline ACI $440.2 \mathrm{R}$ & 32 & 1,11 & 18,39 & $16,53 \%$ & $6,25 \%$ \\
\hline fib Bulletin 14 & 41 & 1,09 & 18,43 & $16,88 \%$ & $7,32 \%$ \\
\hline \multicolumn{6}{|c|}{ Grupo RF } \\
\hline Recomendações & $\begin{array}{c}\text { Qt. de } \\
\text { espécimes }\end{array}$ & Média & $\begin{array}{c}\text { Desvio Padrão } \\
(\%)\end{array}$ & $\begin{array}{l}\text { Coeficiente de } \\
\text { Variação }(\%) \\
\end{array}$ & $\begin{array}{c}\text { Resultados Contra a } \\
\text { Segurança }\end{array}$ \\
\hline ACI $440.2 \mathrm{R}$ & 2 & 1,03 & 2,96 & $2,88 \%$ & $0,00 \%$ \\
\hline fib Bulletin 14 & 2 & 1,04 & 3,00 & $2,88 \%$ & $0,00 \%$ \\
\hline
\end{tabular}

A partir desta análise percebe-se que os valores de desvio padrão e coeficiente de variação reduziram consideravelmente se comparados aos valores obtidos na análise geral do banco de dados. Além disso, as médias obtidas mantiveram-se similares nos três grupos analisados, sempre superiores a 1 e com resultados contra a segurança sempre inferiores à $8 \%$.

Tratando mais especificamente do grupo RF, observa-se que este apresentou valores muito reduzidos de desvio padrão e coeficiente de variação, além de não apresentar nenhum resultado contra a segurança. Porém, devido ao número reduzido de espécimes deste grupo, não se pode afirmar que as recomendações de cálculo sejam mais adequadas para vigas que apresentem este modo de ruptura, uma vez que não existem dados suficientes para tirar conclusões acerca do comportamento das vigas com este tipo de ruptura.

Já os demais grupos apresentam quantidades mais expressivas de resultados, sendo que a grande maioria deles se enquadrou no grupo DE/FL. Este seria o modo de ruptura desejado para os espécimes analisados, visto que as vigas 
romperiam de forma dúctil, por escoamento excessivo do aço ou descolamento das lâminas, evitando a ruptura frágil dada por esmagamento do concreto.

\section{CONCLUSÕES}

Ao coletar o banco de dados e avaliar os resultados apresentados pelos 49 espécimes estudados pelos 12 autores distintos, observa-se que a técnica de reforço NSM mostra-se, de fato, eficaz para aumentar a capacidade resistiva de vigas de concreto armado.

Em relação às prescrições internacionais abordadas neste trabalho, ou seja, o ACI 440.2R (2017) e o fib Bulletin 14 (2001), foi possível observar que, em quesitos de precisão e acurácia, os dois apresentaram previsões razoáveis para o momento último resistente, com valor de R2 superior à 0,9 , desvio padrão em torno de $17 \%$ e coeficiente de variação em torno de $15 \%$, para ambas as prescrições.

Já em relação à análise dos modos de ruptura, observou-se que as previsões foram muito mais adequadas para a norma americana do que para a recomendação europeia, visto que apresentaram uma conformidade de previsão dos modos de ruptura de 69,39\% para o ACI 440.2R (2017) e de 55,10\% para o fib Bulletin 14 (2001). Vale ainda destacar que para o modo de ruptura DE/FL as previsões do modo de ruptura foram excelentes, com taxas de conformidade superiores à $90 \%$ para as duas prescrições. Ressalta-se ainda que para o modo de ruptura CC, ambos modelos teóricos apresentaram taxas de conformidades baixas.

\section{AGRADECIMENTOS}

Os autores gostariam de agradecer pelo apoio a esta e a outras pesquisas à: Universidade de Brasília; ao Laboratório de Estruturas da Universidade de Brasília; ao GETEC-UnB - Grupo de Estudos de Tecnologias da Engenharia Civil; às Agências de fomento FAPDF, CNPq e CAPES.

\section{REFERÊNCIAS BIBLIOGRÁFICAS}

AMERICAN CONCRETE INSTITUTE. ACI 318 - Building Code Requirements for Structural Concrete and Commentary. Farmington Hills, EUA, 2011.

AMERICAN CONCRETE INSTITUTE. ACI 440.2R. Guide for the design and construction of externally bonded FRP systems for strengthening concrete structures. Detroit, EUA, 2015.

BARROS, J.A.O.; DIAS, S.J.E.; LIMA, J.L.T. Efficacy of CFRP- based techniques for the flexural and shear strengthening of concrete beams. J. Cem. Concrete Composites. 29 203-217, Portugal, 2007.

BILOTTA, A.; CERONI, F.; NIGRO, E.; PECCE, M. Efficiency of CFRP NSM strips and EBR plates for flexural strengthening of RC beams and loading pattern influence. Composite Structures, 2014.

COELHO, M.; SILVA, L.; SENA-CRUZ, J.M.; BARROS, J. Estudo comparativo de diferentes técnicas no reforço à flexão de vigas de betão armado com recurso a CFRP's sob acções monotónicas e de fadiga. Revista Portuguesa de Engenharia de Estruturas, Portugal, 2011.

DIAS, S.J.E.; BARROS, J.A.O.; AZEVEDO, C. Comportamento de vigas de betão armado reforçadas à flexão com laminados de CFRP inseridos. Encontro Nacional Betão Estrutural 2012, Lisboa, Portugal, 2012.

FÉDERATION INTERNATIONALE DU BÉTON. fib Bulletin 14. Externally Bonded FRP Reinforcement for RC Structures. Lausanne, Suíça, 2001.

FORTES, A.S.; BARROS, J.A.O. Desempenho de três técnicas de reforço à flexão de vigas de betão armado com CFRP. Encontro Nacional Betão Estrutural 2002, Lisboa, Portugal, pp. 233-242, 2002.

IBRAHIM, W.; FATTAH, W.A.; KOTB, A.; MJEED, M.A. Flexural behavior of RC beams strengthened with CFRP strips. The 7th International Conference on FRP Composites in Civil Engineering, CICE 2014, August 20-22, 
Vancouver, Canadá, 6 pp, 2014.

KANG, J.Y.; PARK, Y.H.; PARK, J.S.; YOU, Y.J.; JUNG, W.T. Analytical evaluation of RC beams strengthened with near surface mounted CFRP laminates. 7th International Symposium, Fiber Reinforced Polymer Reinforcement for Concrete Structures (FRPRCS7), Cidade do Kansas, Estados Unidos, pp. 779- 794, 2005.

KIM, Y.J.; HMIDAN, A.; YAZDANI, S. Variable Shear Span-Depth Ratios for Reinforced Concrete Beams Strengthened with Various Carbon Fiber-Reinforced Polymer Configurations. ACI Structural Journal, Vol. 112, No. $5,2015$.

LIM, D.H. An Experimental Study of Flexural Strengthening Method of Reinforced Concrete Beams with Near Surface Mounted CFRP Strips. Journal of the Korea Society of Civil Engineers, Vol. 33, No. 1, p.131-136, 2013.

MACHADO, A. P.; MACHADO, B. A. Reforço de estruturas de concreto armado com sistemas compostos FRP: teoria e prática. São Paulo: Editora Pini, 2015.

MOON, D.Y.; OH, H.S.; ZI, G.S. Assessment of Flexural Strengthening Behavior Using the Stirrup-Cutting Near Surface Mounted (CNSM) CFRP strip. Journal of the Korea Institute for Structural Maintenance and Inspection, Vol. 16, No. 6, pp.102-112, 2005.

SEO, S.; CHOI, K.; KWON, Y.; LEE, K. Flexural Strength of RC Beam Strengthened by Partially De-bonded Near Surface-Mounted FRP Strip. International Journal of Concrete Structures and Materials Vol.10, No.2, p.149-161, 2016.

YOST, J.R.; GROSS, S.P.; DINEHART, D.W.; MILDENBERG, J.J. Flexural Behavior of Concrete Beams Strengthened with Near-Surface-Mounted CFRP Strips. ACI Structural Journal, Vol. 104, No. 4, 2007 